\title{
Comparative evaluation of the tendon-bone interface contact pressure in different single- versus double-row suture anchor repair techniques
}

\author{
Mike H. Baums · G. Spahn · H. Steckel · A. Fischer • \\ W. Schultz $\cdot$ H.-M. Klinger
}

Received: 15 October 2008/Accepted: 20 February 2009/Published online: 21 March 2009

(C) The Author(s) 2009. This article is published with open access at Springerlink.com

\begin{abstract}
The aim of the study was to evaluate the time zero contact pressure over a defined rotator cuff footprint using different repair and stitch techniques in an established sheep model. Forty fresh-frozen sheep shoulders were randomly assigned to five repair groups: single-row repair using simple stitches (SRA-s), single-row repair using horizontal mattress stitches (SRA-m), and single-row repair using arthroscopic Mason-Allen stitches (SRAama). Double-row repair was either performed with a combination of simple and horizontal mattress stitches (DRA-sm) or with arthroscopic Mason-Allen/horizontal mattress stitches (DRA-amam). Investigations were performed using a pressure-sensitive film system. The average contact pressure and pressure pattern were measured for each group. Contact pressure was lowest in SRA-m followed by SRA-s. SRA-ama showed highest contact pressure of all single-row treatment groups $(P<0.05)$. DRA-amam presented the highest overall contact pressure $(P<0.05)$, whereas DRA-sm exerted contact pressure equal to that of SRA-ama. Both double-row techniques showed the most expanded pressure pattern. Average contact pressures for the more complex single- and doublerow techniques utilizing arthroscopic Mason-Allen stitches
\end{abstract}

M. H. Baums $(\bowtie)$ · A. Fischer - W. Schultz · H.-M. Klinger Department of Orthopaedic Surgery, University of Göttingen Medical Centre (UMG), Georg-August-University, Postfach, 37099 Göttingen, Germany

e-mail: mbaums@t-online.de

\section{G. Spahn}

Clinic of Orthopaedic Surgery and Traumatology Eisenach,

Eisenach, Germany

H. Steckel

Department of Orthopaedic Surgery, Park-Klinik Weissensee, Berlin, Germany were greater than were those of the repair techniques utilizing simple and horizontal mattress stitches. However, the contact pattern between the anchors could be increased by using the double-row technique, resulting in more footprint coverage compared to patterns utilizing the single-row techniques. These results support the use of the more complex arthroscopic Mason-Allen stitches and may improve the environment for healing of the repaired rotator cuff tendon.

Keywords Rotator cuff repair - Contact pressure . Double-row · Single-row · Sheep shoulder

\section{Introduction}

The repair of the rotator cuff remains a challenge, because structural failure and recurrent tears are frequent postoperative problems. Based on ultrasound and MRI evaluation, re-tear rates for arthroscopic rotator cuff repair after massive rotator cuff injury have been reported to range from 30 to $94 \%[3,4,9]$. This implies that current techniques fail to establish an adequate environment to facilitate healing of the tendon to the bone. The development of a fibrovascular interface between the tendon and the bone is the primary requirement for sufficient healing. With such an interface, it is possible to restore fibrocartilagenous tendon insertion [14]. It is postulated that re-establishing the anatomical configuration of the tendon-bone insertion is a key factor in optimization of this process [1]. Although most techniques may not adequately restore the native footprint area the double-row technique allows nearly 100\% coverage [1]. However, the excellent initial mechanical strength and footprint coverage of the double-row technique do not guarantee clinical superiority $[6,8]$. These studies show 
that merely covering the anatomical footprint area does not lead to better results compared to alternative repair techniques, e.g. a single-row of anchors. Therefore, it must be determined whether there is a repair technique that achieves sufficient contact pressure to minimize motion between the tendon and its insertion site, thus optimizing the healing process.

In light of this, the objective of our study was to determine the initial contact pressure over a defined rotator cuff footprint using different arthroscopic repair and stitch techniques in an established animal model. We hypothesized that arthroscopic Mason-Allen stitches in a singlerow configuration would result in similar contact pressure to that yielded by a double-row repair using a combination of arthroscopic Mason-Allen and horizontal mattress stitches.

\section{Materials and methods}

Forty sheep shoulders (specimen age, 2 years) were freshly harvested, wrapped in saline-soaked gauze, and stored frozen at $-20^{\circ} \mathrm{C}[2,15,30]$. Shoulders were dissected from all of the soft tissues except for the infraspinatus muscle and tendon. No pre-existing rotator cuff abnormalities were noted in any of the specimens. The infraspinatus tendon was sharply detached from its insertion site to mimic a fullthickness tear, as established in previous investigations at our institution [2, 15]. Right and left shoulders were randomly assigned among five treatment groups. We used nonabsorbable suture anchors (SUPER REVO ${ }^{\circledR}$ Suture Anchor, ConMed Linvatec, Largo, FL) coupled with braided nonabsorbable polyester suture sized USP No. 2 (Ethibond $^{\circledR}$; Ethicon, Sommerville, NJ). The groups were as follows (Fig. 1a-e):

- Single-row anchor-simple (SRA-s): simple stitch repair using one line of two double-loaded suture anchors.

- Single-row anchor-mattress (SRA-m): horizontal mattress stitch repair using one line of two double-loaded suture anchors.

- Single-row anchor-arthroscopic Mason-Allen (SRAama): arthroscopic Mason-Allen stitch repair using one line of two double-loaded suture anchors.

- Double-row anchor-simple mattress (DRA-sm): a combination of simple (lateral/double-loaded) and horizontal mattress stitches (medial/single-loaded) repair using two lines with a total of four suture anchors.

- Double-row anchor-arthroscopic Mason-Allen mattress (DRA-amam): a combination of arthroscopic MasonAllen (lateral/double-loaded) and horizontal mattress stitches (medial/single-loaded) repair using two lines with a total of four suture anchors.

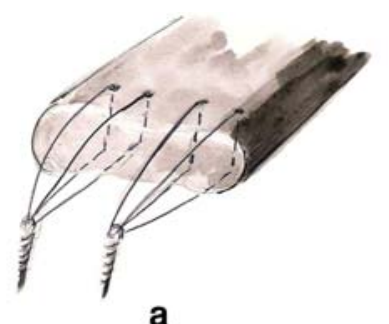

a
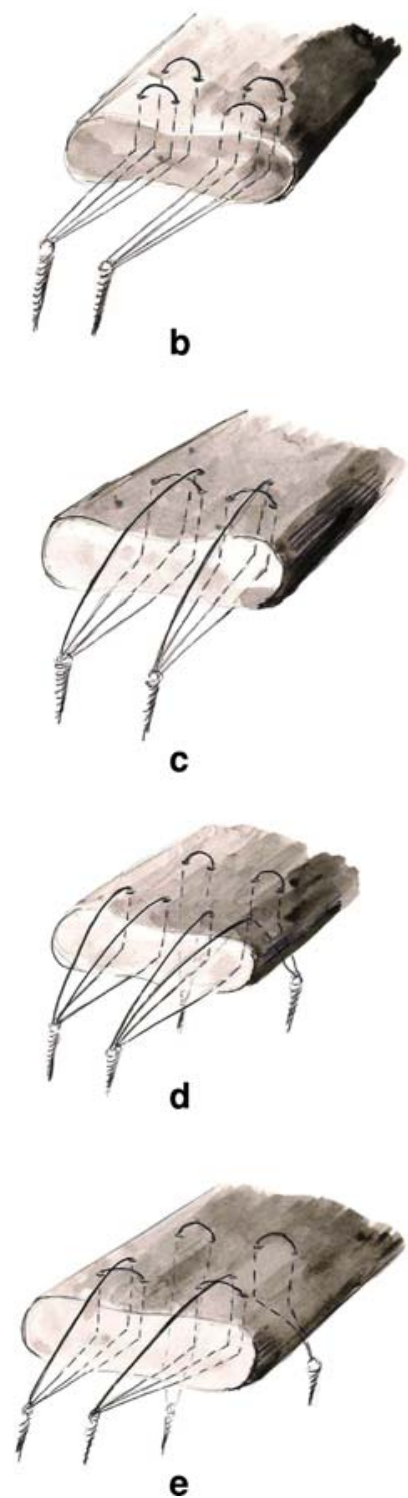

Fig. 1 Schematic line drawing of the used repair techniques. a One line of two double-loaded suture anchors using simple stitches (SRAs). b One line of two double-loaded suture anchors using horizontal mattress stitches (SRA-m). c One line of two double-loaded suture anchors using arthroscopic Mason-Allen stitches (SRA-ama). d Two lines with a total of four suture anchors using a combination of simple (lateral/double-loaded) and horizontal mattress stitches (medial/ single-loaded) (DRA-sm). e Two lines with a total of four suture anchors using a combination of arthroscopic Mason-Allen (lateral/ double-loaded) and horizontal mattress stitches (medial/singleloaded) (DRA-amam) 
In a pilot study the footprint area of the infraspinatus tendon averaged $1 \times 2 \mathrm{~cm}$. Therefore, a template was prepared beforehand to facilitate standardized insertion of the suture anchor systems. After using a cannulated tap to prepare the bone each anchor system was placed over a guidewire according to the manufacturer guidelines. Then, the anchor was inserted into the bone at a $45^{\circ}$ angle where the eyelets were flush with the bone [5]. Afterwards the tendon was reattached to its insertion site. No pretension was applied to the tendon during repair. To simulate an arthroscopic setting all anchor system insertion steps, as well as suture passing, were performed with instruments for arthroscopic repair (Spectrum tissue repair system, ConMed Linvatec, Largo, FL) [2]. In addition, the procedure was performed utilizing an arthroscopic knot-tying technique with use of an arthroscopic knot pusher. Each stitch was first tied with a sliding double half-hitch knot, secured by a series of four reversing half-hitches on alternative posts. To standardize tension for the repair, no less than $4 \mathrm{~kg}$ of tensile force was used to secure each knot. This was measured by a tensiometer. The dissections, preparations, and repairs were performed by a single experienced shoulder surgeon (MHB) after thawing the shoulders for $24 \mathrm{~h}$ at room temperature. Standard procedures for rotator cuff repairs were used to minimize technical variability.

\section{Experimental testing}

The investigations were performed at room temperature using a pressure-sensitive film system (Prescale film, Super Low Pressure type, Fuji Photo Film Co Ltd, Tokyo, Japan). This allowed measurement of the interface contact pattern as well as of the contact pressure between the infraspinatus tendon and the insertion site. The used Prescale film is two-sheet (composed of an A- and C-film) and detects a pressure range between 0.50 and $2.50 \mathrm{MPa}$. The A-film is coated with micro-encapsulated colourforming material, while the C-film is coated with colourdeveloping material. When pressure is applied, the microcapsules break and the colour-forming material reacts with the colour-developing material to generate colour. The microcapsules are designed to react incrementally to the level of pressure; thus the colour density corresponds to the level of pressure.

The pressure-sensitive film was cut in a standardized fashion for all specimens to conform to the $1 \times 2 \mathrm{~cm}$ footprint. Then, the film was placed under the prepared template so that we could prepare uniform and symmetric holes on both film sheets. The pressure sensitive film was sealed in an impermeable polyethylene sheet. After insertion of the suture anchor systems, the film was inserted between the tendon-bone interfaces. We kept the film dry by continually using gauze to absorb the moisture from the tendon and the bone. The sutures connecting tendon to bone were carefully passed through the prepared holes to allow the best possible panoramic view of the contact pressure and pressure pattern. After the repair, the film was left in place for $2 \mathrm{~min}$, as recommended by the manufacturer. Sutures were then carefully cut, and the film was scanned with a Fuji Film Prescale Pressure Densitometer (FDP-305E, Fuji Photo Film Co. Ltd). The average contact pressure and pressure pattern were recorded.

\section{Statistical analysis}

Statistical analysis was performed using SPSS software (Rel. 13.0, SPSS, Chicago, IL). The average contact pressure was subjected to analysis of variance (ANOVA). Results are reported as mean values \pm standard deviation. The level of significance was set at $P<0.05$.

\section{Results}

As expected, the double-row techniques (DRA-sm, DRAamam) maintained better contact with the insertion area than the single-row techniques (SRA-s, SRA-m, SRAama). However, the pressure around the insertion of each anchor system was higher than in the area between the anchor systems (Fig. 2a, b). The infraspinatus tendon using the single-row simple stitch repair consisting of one line of two double-loaded suture anchors (SRA-s) resulted in a mean contact pressure of $1.07 \pm 0.11 \mathrm{MPa}$ applied on the footprint area. On the other hand, the single-row repair performed with horizontal mattress stitches (SRA-m) exerted the lowest contact pressure on the footprint $(0.95 \pm 0.09 \mathrm{MPa})$. The highest contact pressure of the single-row techniques used was $1.15 \pm 0.05 \mathrm{MPa}$ exerted by the arthroscopic Mason-Allen stitches (SRA-ama). In comparison, the contact pressures between SRA-s and SRA-m were not statistically different $(P>0.05)$. The use of arthroscopic Mason-Allen stitches (SRA-ama) produced the highest contact pressure of all single-row techniques $(P<0.05)$.

The double-row repair, utilizing two lines with a total of four suture anchors using a combination of simple and horizontal mattress stitches (DRA-sm) produced a contact pressure of $1.15 \pm 0.03 \mathrm{MPa}$ over the footprint. The double-row technique performed with a combination of arthroscopic Mason-Allen and horizontal mattress stitches (DRA-amam) applied a contact pressure of $1.19 \pm$ $0.03 \mathrm{MPa}(P<0.05)$. The DRA-amam exerted a significantly higher contact pressure compared to the SRA-ama $(P<0.05)$. Results are summarised in Fig. 3 . 
a
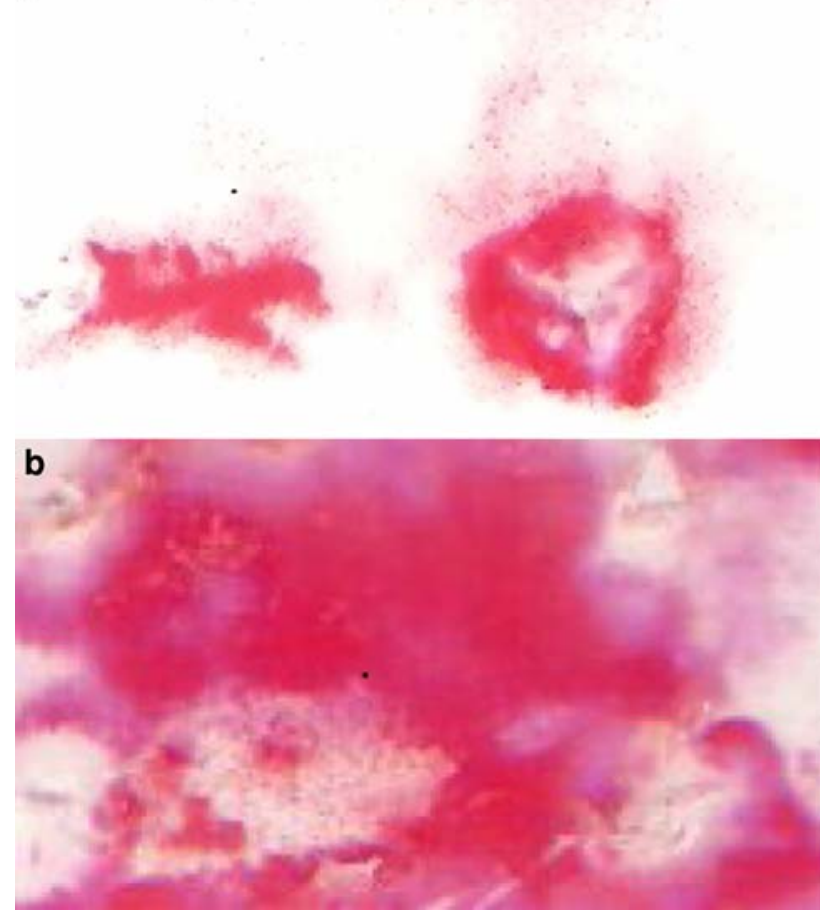

Fig. 2 Typical pressure pattern of a (a) single- (SRA-ama) and (b) double-row (DRA-amam) repair. Contact pattern between the anchors could be increased by the double-row technique, resulting in more footprint coverage. The pressure around the insertion of each anchor system was higher than in the area between the anchor systems as represented by the colour density

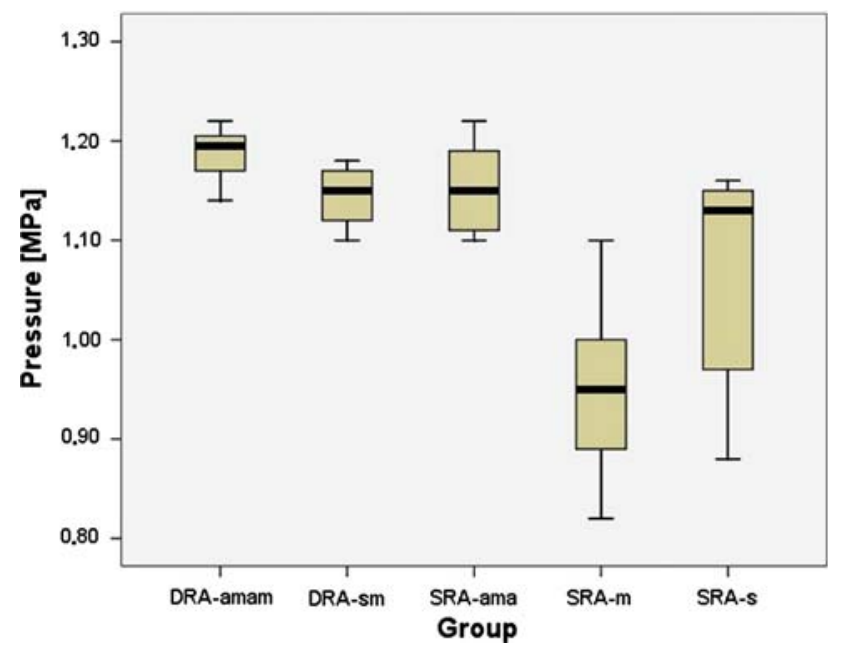

Fig. 3 Mean pressure distributions of the evaluated repair techniques. Contact pressure was lowest in SRA-m and SRA-s. SRA-ama showed highest contact pressure of all single-row treatment groups $(P<0.05)$. DRA-amam presented the highest overall contact pressure $(P<0.05)$, whereas DRA-sm exerted contact pressure equal to that of SRA-ama $(P>0.05)$

\section{Discussion}

The most important finding of the present study was that the double-row repair techniques exerted the highest contact pressure. We did not confirm our hypothesis that double-row repair using a combination of arthroscopic Mason-Allen and horizontal mattress stitches (DRAamam) would provide a similar contact pressure to arthroscopic Mason-Allen stitches in a single-row configuration (SRA-ama). However, the outcome for SRA-ama $(1.15 \pm 0.05 \mathrm{MPa})$ was similar to that obtained for DRAsm $(1.15 \pm 0.03 \mathrm{MPa})(P>0.05)$.

Recent studies have documented the mechanical characteristics of several rotator cuff repair techniques [2, 13$20,22,23,26]$ with excellent results for the double-row repair $[2,16,18,19]$. Nonetheless, its clinical superiority remains to be proven. A prospective MRI follow-up evaluation failed to reveal any significant differences between double- and single-row techniques [6], whereas a computed tomographic arthrography investigation demonstrated better tendon healing results of the double-row repair[8]. In addition, the results of clinical assessments were similar to the single- and double-row groups. Cummins et al. [7] showed that not only the number of suture anchor systems, but also the configuration of the sutures in the soft tissue, is an important determinant in the success or failure of the repair. Arthroscopic techniques for rotator cuff repair usually rely upon easier-to-perform simple (Fig. 1a) or horizontal mattress stitches (Fig. 1b) fixed with suture anchors. However, these stitches prevent formation of a firm tendon-bone construct, especially in comparison to the modified Mason-Allen transosseous suture technique [7]. Scheibel and Habermeyer [25] performed an arthroscopic Mason-Allen technique for suture anchor repair. The technique consists of horizontal mattress and single stitches travelling through the same anchor (Fig. 1c). In a recent study, we demonstrated the superior strength of this stitch technique compared to the modified Mason-Allen transosseous suture technique [15].

Several studies have demonstrated the influence of tendon-to-bone interface pressure on tendon biology, especially for anterior cruciate ligament reconstruction [11, $12,29]$. Our review of the literature revealed few papers examining the contact pressures exerted by different rotator cuff repair techniques [20-22, 27]. In a recent study, Nelson et al. [20] found that a double-row repair using a medial row mattress suture and a lateral row simple suture configuration yields a significantly larger repair area compared to a single-row repair using arthroscopic MasonAllen stitches. Contact pressure was not evaluated in this study.

Park et al. [22] assigned 25 fresh-frozen bovine shoulders to three repair techniques: (1) a transosseous tunnel 
simple suture technique, (2) a suture anchor technique performed with simple stitches, (3) and a suture anchor technique performed with mattress stitches. The transosseous tunnel technique created significantly more contact and overall pressure distribution over a defined footprint compared to both of the suture anchor techniques. The authors hypothesized that stronger and faster rotator cuff healing may be expected for the best possible pressure distributions of the repaired tendon-bone interface. In contrast, Tuoheti et al. [27] showed that double-row repair in ten cadaveric shoulder specimens created the greatest contact area and second-highest contact pressure, whereas the transosseous technique produced the second-greatest footprint but the least contact pressure. Single-row repair showed the highest contact pressure and the least contact area. The authors concluded that the double-row suture anchor repair and the transosseous technique may provide a better environment for tendon healing compared to the single-row repair. Park et al. [21] demonstrated that a 4-suture bridge technique yielded a significantly higher contact area and interface pressure than double-row and 2 -suture-bridge techniques. The suture-bridge technique is transosseous-equivalent and consists of a medial row of anchor systems. The repair is secured by placing drill holes and fixed with suture bridges. The authors conclude that this technique may lead to further improvement in the repair of rotator cuff tears.

Despite the importance of stitch techniques for mechanical strength of the repaired tendon-bone interface, no study had investigated the contact pressure and pressure patterns with consideration of commonly used stitch techniques. Our study shows that, in combination with arthroscopic Mason-Allen stitches, the average contact pressures of the more complex single- and double-row techniques were greater than those of repair techniques using simple and horizontal mattress stitches. On the other hand, the contact pattern between the anchors could be increased by the double-row technique, resulting in more footprint coverage than is observed for single-row techniques [27].

Our investigation presents some weaknesses only visible in application of these theoretical models to practical situations. Although our animal model is well established for research of rotator cuff repair techniques [2, 10, 14, 15], it remains an approximation to the human condition and clinically used arthroscopic repair techniques. But due to space limitations inherent to the anatomy of a sheep shoulder an all-arthroscopic repair of the rotator cuff is nearly impossible. Therefore, the reconstructions were done in an open fashion with use of instruments for arthroscopic repair. In addition, we did not analyse the surface area but the contact pressure between the tendon and the bone lone.
Furthermore, although great care was taken to minimize interference when preparing holes in the film, the process may have yielded some artefacts. However, it would be impossible to obtain the entire contact pressure distribution and pressure pattern of the investigated techniques if pressure-sensitive film was only inserted between the tendon-bone interface, particularly with regard to the different stitch techniques. Using pressure-sensitive film that detects a pressure range between 0.50 and $2.50 \mathrm{MPa}$ may have underestimated the contact area [27].

The double-row technique displayed excellent initial mechanical strength $[2,13,16,18,19]$ and pressure distribution [27] but demands critical discussion. It is a more complex method and requires more surgical time compared to single-row of treatment. It requires careful suture management and a safe command level, especially for arthroscopic repair, and produces more costs in suture anchor material. Additionally, the results of our study show an almost equal contact pressure when comparing the more complex arthroscopic Mason-Allen stitch technique in a single-row configuration (SRA-ama) to a double-row configuration utilizing simple/mattress stitches (DRA-sm). Beyond this consideration we do not know the optimal pressure range for the tendon to heal to the bone. In particular, the depth of anchor placement and thickness of the tendon appear to determine the amount of compression that a suture anchor system can provide [21]. Therefore, low pressure may cause separation of the tendon-bone interface, whereas high pressure may affect the vascularisation of the tendon and result in impaired tissue healing. Thus, the mechanical strength and suture anchor placement of a double-row repair may produce more tension on the tendon and impact vascularisation.

Considering the outcome of recent clinical and basic trials, further research is necessary to optimize restorations of the rotator cuff footprint and to achieve lower failure rates. In particular, the widely used suture bridge technique will be evaluated in further studies. Successful rotator cuff surgery demands enormous costs [24, 28]; failure increases costs exponentially. The double-row repair will be more expensive than previous repair techniques and currently garner equivalent clinical outcomes [6, 8]. In an effort to maximize success of these new techniques, it would be beneficial to measure the real-time pressure distributions because we do not know whether the contact pressure and contact pattern may change under dynamic loading conditions.

\section{Conclusion}

In conclusion, the fundamental results of our study support the use of more complex stitch techniques (e.g. arthroscopic Mason-Allen stitches) as opposed to simple/mattress 
stitches in order to obtain higher contact pressure of the tendon on the footprint surface, thus optimizing the healing process. Due to biomechanical characteristics, the doublerow technique combined with this stitch configuration may provide an environment conducive to the primary stability of this repair [2]. We anticipate the results of further in vivo investigations regarding cell biological characteristics and the extent to which the conclusions of our ex vivo study may contribute to improved healing of the repaired rotator cuff.

Acknowledgments The work was founded by the Research Program, University of Göttingen Medical Centre (UMG), GeorgAugust-University, Germany. The authors acknowledge Mrs. G. Wolf-Bergk for creating the anatomical line drawings in this report.

Open Access This article is distributed under the terms of the Creative Commons Attribution Noncommercial License which permits any noncommercial use, distribution, and reproduction in any medium, provided the original author(s) and source are credited.

\section{References}

1. Apreleva M, Özbaydar M, Fitzgibbons PG, Warner JJP (2002) Rotator cuff tears: the effect of the reconstruction method on three-dimensional repair site area. Arthroscopy 18:519-526

2. Baums MH, Buchhorn GH, Spahn G, Poppendieck B, Schultz W, Klinger HM (2008) Biomechanical characteristics of single-row repair in comparison to double-row repair with consideration of the suture configuration and suture material. Knee Surg Sports Traumatol Arthrosc 16:1052-1060

3. Bishop J, Klepps S, Lo IK, Bird J, Gladstone JN, Flatow EL (2006) Cuff integrity after arthroscopic versus open rotator cuff repair: a prospective study. J Shoulder Elbow Surg 15:290-299

4. Boileau P, Brassart N, Watkinson DJ, Carles M, Hatzidakis AM, Krishnan SG (2005) Arthroscopic repair of full-thickness tears of the supraspinatus: does the tendon really heal? J Bone Joint Surg Am 87-A:1229-1240

5. Burkhart SS (1995) The deadman theory of suture anchors: observations along a south Texas fence line. Arthroscopy 11:119-123

6. Charousset C, Grimberg J, Duranthon LD, Bellaiche L, Petrover D (2007) Can a double-row anchorage technique improve tendon healing in arthroscopic rotator cuff repair? A prospective, nonrandomized, comparative study of double-row and single-row anchorage techniques with computed tomography arthrography tendon healing assessment. Am J Sports Med 35:1247-1253

7. Cummins CA, Murrell GA (2003) Mode of failure for rotator cuff repair with suture anchors identified at revision surgery. J Shoulder Elbow Surg 12:128-133

8. Franceschi F, Ruzzino L, Longo UG, Martina FM, Zobel BB (2007) Equivalent clinical results of arthroscopic single-row and double-row suture anchor repair for rotator cuff tears. Am J Sports Med 35:1254-1260

9. Galatz LM, Ball CM, Teefey SA, Middleton WD, Yamaguchi K (2004) The outcome and repair integrity of completely arthroscopically repaired large and massive rotator cuff tears. J Bone Joint Surg Am 86-A:219-224

10. Gerber C, Schneeberger AG, Beck M, Schlegel U (1994) Mechanical strength of repairs of the rotator cuff. J Bone Joint Surg Br 76-B:371-380
11. Goradia VK, Rochat MC, Kida M, Grana WA (2000) Natural history of hamstring tendon autograft used for anterior cruciate ligament reconstruction in a sheep model. Am J Sports Med 28:40-46

12. Katsuragi R, Yasuda K, Tsujino J, Keira M, Kaneda K (2000) The effect of nonphysiologically high initial tension on the mechanical properties of in situ frozen anterior cruciate ligament in a canine model. Am J Sports Med 28:47-56

13. Kim DH, ElAttrache NS, Tibone JE, Jun BJ, DeLaMora SN, Kvitne RS, Lee TQ (2005) Biomechanical comparison of a single-row versus double-row suture anchor technique for rotator cuff repair. Am J Sports Med 34:407-414

14. Klinger HM, Koelling S, Baums MH, Kahl E, Steckel H, Smith MM, Schultz W, Miosge N (2008) Cell biological and biomechanical evaluation of two different fixation techniques for rotator cuff repair. Scand J Med Sci Sports Apr 6 (Epub ahead of print). doi:10.1111/j.1600-0838.2008.00791.x

15. Klinger HM, Steckel H, Spahn G, Buchhorn GH, Baums MH (2007) Biomechanical comparison of double-loaded suture anchors using arthroscopic Mason-Allen stitches versus traditional transosseous suture technique and modified Mason-Allen stitches for rotator cuff repair. Clin Biomech 22:106-111

16. Ma CM, Comerford L, Wilson J, Puttlitz CM (2006) Biomechanical evaluation of arthroscopic rotator cuff repairs: doublerow compared with single-row fixation. J Bone Joint Surg Am 88-A:403-410

17. Mahar A, Tamborlane J, Oka R, Esch J, Pedowitz RA (2007) Single-row suture anchor repair of the rotator cuff is biomechanically equivalent to double-row repair in a bovine model. Arthroscopy 23:1265-1270

18. Meier SW, Meier JD (2006) The effect of double-row fixation on initial repair strength in rotator cuff repair: a biomechanical study. Arthroscopy 22:1168-1173

19. Milano G, Grasso A, Zarelli D, Deriu L, Cillo M, Fabbriciani C (2008) Comparison between single-row and double-row rotator cuff repair: a biomechanical study. Knee Surg Sports Traumatol Arthrosc 16:75-80

20. Nelson CO, Sileo MJ, Grossman MG, Serra-Hsu F (2008) Singlerow modified Mason-Allen versus double-row arthroscopic rotator cuff repair: a biomechanical and surface area comparison. Arthroscopy 24:941-948

21. Park MC, ElAttrache NS, Tibone JE, Ahmad CS, Jun BJ, Lee TQ (2007) Part I: Footprint contact characteristics for a transosseousequivalent rotator cuff repair technique compared with a doublerow technique. J Shoulder Elbow Surg 16:461-468

22. Park MC, Cadet ER, Levine WN, Bigliani LU, Ahmad CS (2005) Tendon-to-bone pressure distributions at a repaired rotator cuff footprint using transosseous suture and suture anchor fixation techniques. Am J Sports Med 33:1154-1159

23. Petit CJ, Boswell R, Mahar A, Tasto J, Pedowitz RA (2003) Biomechanical evaluation of a new technique for rotator cuff repair. Am J Sports Med 31:849-853

24. Savoie FH 3rd, Field LD, Jenkins RN (1995) Cost analysis of successful rotator cuff surgery: an outcome study. A comparison of gatekeeper system in surgical patients. Arthroscopy 11:672676

25. Scheibel MT, Habermeyer P (2003) A modified Mason-Allen technique for rotator cuff repair using suture anchors. Arthroscopy 19:330-333

26. Schneeberger AG, von Roll A, Kalberer F, Jacob HA, Gerber C (2002) Mechanical strength of arthroscopic rotator cuff repair techniques: an in vitro study. J Bone Joint Surg Am 84-A:21522160

27. Tuoheti Y, Itoi E, Yamamoto N, Seki N, Abe H, Minagawa H, Okada K, Shimada Y (2005) Contact area, contact pressure, and 
pressure patterns of the tendon-bone interface after rotator cuff repair. Am J Sports Med 33:1869-1874

28. Vitale MA, Vitale MG, Zivin JG, Braman JP, Bigliani LU, Flatow EL (2007) Rotator cuff repair: an analysis of utility scores and cost-effectiveness. J Shoulder Elbow Surg 16:181-187

29. Weiler A, Hoffmann RFG, Bail HJ, Rehm O, Südkamp NP (2002) Tendon healing in a bone tunnel, part II: histologic analysis after biodegradable interference fir fixation in a model of anterior cruciate ligament reconstruction in sheep. Arthroscopy 18:124-135

30. Woo SL, Orlando CA, Camp JF, Akeson WH (1986) Effects of postmortem storage by freezing on ligament tensile behaviour. J Biomech 19:399-404 